\title{
Emergence delirium and intranasal dexmedetomidine premedication in pediatric anesthesia: a retrospective study in plastic surgery
}

Alessandra Di Palma ( $\square$ submission@polistudium.it)

Ospedale Pediatrico Bambino Gesu

Federica Maldarelli

Universita degli Studi di Roma La Sapienza

Antonietta Cimino

Ospedale Pediatrico Bambino Gesu

Mario Zama

Ospedale Pediatrico Bambino Gesu

Sergio Giuseppe Picardo

Ospedale Pediatrico Bambino Gesu

Research article

Keywords: Emergency delirium, Dexmedetomidine, Anesthesia

Posted Date: December 18th, 2019

DOI: https://doi.org/10.21203/rs.2.16196/v2

License: (c) (1) This work is licensed under a Creative Commons Attribution 4.0 International License.

Read Full License 


\section{Abstract}

\section{Background}

Dexmedetomidine is increasingly used in the treatment of emergency delirium (ED) in pediatric patients. However, further evidence on its use in pediatric anesthesia and on potential differences in the reduction of ED according to patient's age and type of anesthesia is required. Moreover, whether dexmedetomidine influences time of discharge from the surgical area remains unclear. We evaluated whether intranasal dexmedetomidine is effective in decreasing the incidence of ED in 106 children who had anesthesia for plastic surgery undergoing general or combined anesthesia at different ages. We also assessed if this drug has an impact on time to discharge from the surgical area.

Methods

In total, 106 children, aged 2-10 years, were enrolled in this retrospective study. Among them, 50 have been premedicated with dexmedetomidine (dexmedetomidine group); the remaining 56 patients served as controls (control group). The incidence of ED was evaluated according to the use of dexmedetomidine premedication, age and type of anesthesia (general vs combined). The length of anesthesia and duration of staying in the surgical area were also analyzed.

Results

Three patients who received dexmedetomidine premedication showed ED (6\%), compared with 43 patients in the control group (77\%; $p<0.05)$. This lower incidence of ED was also present when stratifying patients according to the type of anesthesia or age. We observed no significant difference between the dexmedetomidine group and control group in terms of discharge from surgical area.

\section{Conclusions}

Premedication with dexmedetomidine is associated with decreased incidence of ED, regardless of patients' age or type of anesthesia and without seemingly increasing the timing of discharge after surgery.

\section{Background}

Recovery from anesthesia is a very delicate moment, especially in children [1]. Indeed, children are particularly prone to the development of a delirium at the time of awakening, defined as "emergency delirium" (ED), with an estimated incidence that can be as high as $80 \%$, although reported incidence largely varies [2,3]. ED is a dissociated state of consciousness in which the child may not be able to recognize family members or common objects. The child with ED appears inconsolable, irritable, noncooperating, with psychomotor agitation, crying or persistent complaining and incoherent or incomprehensible language skills. Although ED episodes usually last from 5 to 15 minutes and resolve spontaneously, they can show great intensity, placing the child at risk of auto-injury. Several studies have 
shown that ED in children may be associated with postoperative behavioral problems that were not present before, including eating disturbances, sleep disorders, aggression, apathy, and anxiety of separating from parents [4-6].

ED seems to be associated with several risk factors, such as age (highest frequency between 2 and 6 years) [7], patient's attitude (children who are more emotional, impulsive and have difficulty in socializing, are more prone to experience ED) [8], type of surgery (major surgery with intraoperative complications and surgery affecting the head and the neck) [9], uncontrolled pain and inhalational anesthesia [1].

Nowadays, the best approach to reduce the risk of postoperative ED is its prevention $[10,11]$.

Dexmedetomidine is a high-affinity $₫ 2$-mimetic drug. In particular, this molecule acts in the locus coeruleus determining a sedation pattern similar to natural sleep, without determining respiratory depression [12-14]. Recent studies suggest, among the remedies against ED, the use of dexmedetomidine intranasally as a premedication $[10,11,15,16]$. In particular, a recent meta-analysis showed that intranasal dexmedetomidine provides more satisfactory sedation and reduces the need for rescue analgesics and the incidence of nasal irritation and postoperative nausea and vomiting compared with other premedication treatments [10]. This molecule now has a well-established role in clinical practice; however, further evidence on its use in pediatric anesthesia, in particular, on potential differences in its efficacy according to the patient's age and type of anesthesia, is required [11]. Moreover, whether dexmedetomidine actually influences time of discharge from the surgical area remains unclear [17].

Therefore, we conducted a proof-of-concept study to evaluate whether dexmedetomidine administered intranasally is effective in decreasing the incidence of ED in children who had anesthesia for plastic surgery at different ages, between 2 and 3 years, between 3 years and 6 years and between 6 and 10 years. We also compared the use of dexmedetomidine in patients undergoing general anesthesia or combined anesthesia, to investigate whether in these patients a decreased incidence of ED can be obtained by reducing the administration of general anesthetics, a known risk factor for ED [1]. Lastly, we assessed if dexmedetomidine has an impact on time to discharge from the surgical area.

\section{Methods}

\section{Setting and design}

This retrospective study was conducted at the IRCCS Ospedale Pediatrico Bambino Gesù, a referral center for the treatment of pediatric patients in Italy, from January 2018 to January 2019. The local Ethical Committee approved the study design (protocol number 1587_OPBG_2018), and the legal guardians of all patients signed an informed consent for the use of the patient's data for research purposes.

\section{Patients}


In total, 106 children of any gender, aged 2-10 years who underwent plastic and reconstructive surgery (first and elective) lasting 60-90 minutes were considered. All patients had to be classified as American Society of Anesthesiologists (ASA) grade I and II; patients were not considered if they presented psychiatric disorders or mental retardation, alterations of metabolism or neurological deficit, or could not receive dexmedetomidine (e.g. due to known intolerance, history of cardiac disease, stroke, heart block, intracranial bleeding, beta-blocker or digitalis therapy).

\section{Procedures}

Patients' treatment and assessment were performed in line with standardized internal protocols used at IRCCS Ospedale Pediatrico Bambino Gesù for the treatment of pediatric patients undergoing plastic or reconstructive surgery.

All patients received anesthesia induction via facemask with increasing concentrations of sevoflurane (max: $8 \%$ ) in $40 \%$ oxygen and $60 \%$ nitrous oxide. When bispectral index (BIS) values were between 50 and 40 , a venous access was acquired, through which $2 \mu \mathrm{g} / \mathrm{kg}$ of fentanyl and $0.6 \mathrm{mg} / \mathrm{kg}$ of rocuronium were administered before intubation. Anesthesia was then maintained with $2.5 \%$ sevoflurane end tidal ( $40 \%$ oxygen and $60 \%$ air). If patients showed signs of pain or discomfort during anesthesia, they were treated with fentanyl $2 \mu \mathrm{g} / \mathrm{kg}$.

All patients received a fluid therapy with physiological saline solution $5-10 \mathrm{ml} / \mathrm{kg} / \mathrm{h}$ and ranitidine 2 $\mathrm{mg} / \mathrm{kg}$ to ensure gastric protection. At awakening, intravenous paracetamol was administered at a dose of $15 \mathrm{mg} / \mathrm{kg}$ and ketorolac $0.5 \mathrm{mg} / \mathrm{kg}$ if required by internal protocols.

Clinical monitoring throughout the course of anesthesia for all patients comprised continuous $\mathrm{ECG}, \mathrm{SpO}_{2}$, temperature, BIS, mean arterial pressure every 3 minutes.

Extubation was performed when patients had a BIS score of 60 ; after extubation patients were transferred to the recovery room (BIS between 60 and 100), where they were monitored for vital signs $\left(\mathrm{SpO}_{2}\right.$, continuous ECG, mean arterial pressure every five minutes and temperature).

Different measures were put in place to decrease patient's distress during this period. Parents were allowed in the recovery room to assist the patient, analgesics (such as ketorolac and paracetamol) were administered if needed to control pain, and patients received glucose solution drops under the tongue to reduce the symptoms of hunger. If patients still showed inconsolable crying, irritability and dissociation, these were considered as signs of ED, and patients were treated with midazolam $0.05 \mathrm{mg} / \mathrm{kg}$.

As per hospital internal procedures, the onset of ED was evaluated during this period using the PAED Scale [18-20].This scale assesses the presence of ED by evaluating different aspects of children's exhibited behavior, such as the ability to maintain a visual contact with those around him, if his/her movements are finalized, his/her level of restlessness and inconsolability; each item is classified as 
"extremely present", "very much present", "quite a bit present", "present just a little" and "not at all present", which correspond to a score ranging from 0 to 4 . The final PAED score, obtained by the sum of all the values, ranges from 0 to 20 , with scores $>12$ that indicate the presence of $\operatorname{ED}[19,20]$. Moreover, as per intra-hospital protocol, to determine whether the child could be discharged, we used the Aldrete scale [21] and we assessed the pain level in the recovery room through the visual analogue scale (VAS; for patients $\geq 4$ years old) or the Face, Legs, Activity, Cry, Consolability (FLACC) scale (for patients $<4$ years old) [22]. Patients were discharged for VAS/FLACC $\leq 4$, Aldrete score $\geq 9$ and PAED $\leq 12$; however discharge always occurred after at least 15 minutes of stay in the recovery room.

\section{Use of dexmedetomidine}

Among the 106 evaluated patients, 50 have been premedicated with dexmedetomidine (dexmedetomidine group), at the dose of $2.5 \mathrm{gg} / \mathrm{kg}$ administered intranasally 30 minutes before anesthesia induction [23]. The decision to administer dexmedetomidine was taken by the anesthesiologist, according to the consideration of the grade of anxiousness of that patient at the moment of the preoperatory visit and according to internal protocols. A nasal mucosal atomization device (LMA MAD Nasal, Teleflex, NC) was used for premedication [24].

Patients who did not receive dexmedetomidine premedication represented the control group $(n=56$; control group).

\section{Evaluations}

We evaluated the incidence of ED according to the use of dexmedetomidine premedication. A subgroup analysis was also performed with respect to the use of general anesthesia or combined anesthesia (general anesthesia and locoregional block that could be brachial plexus block, tap block, sciatic and femoral nerve block or penile nerve block). Patients were also stratified within each group according to age (2-3 years, 3-6 years, 6-10 years) to investigate the potential effects of age on the incidence of ED.

The length of anesthesia and duration of staying in the surgical area were also evaluated to analyze whether the premedication with dexmedetomidine could affect these parameters. Hemodynamic parameters (heart rate, mean arterial pressure) were also monitored, and any adverse event was recorded and classified based on the Common Terminology Criteria for Adverse Events (CTCAE), version 4.0; the potential association of adverse events with dexmedetomidine was judged by the treating physicians.

\section{Statistical analysis}

Our sample size estimation was based on the incidence of ED in children who received preoperative intranasal dexmedetomidine and in children who received only saline solution [16] In total, 27 patients 
were required in each group to detect a $45 \%$ difference in the incidence of ED between the groups with a statistical power of $90 \%$ and a type I error a equal to $1 \%$. Considering a dropout rate of $20 \%$, the minimum number of patients to include was increased to 34 per group.

Data were analyzed by descriptive statistics. Patient's characteristics were compared by Student's T test or Pearson's Chi-square test, when appropriate. The incidence of ED and the hemodynamic parameters, statistical comparisons among groups were performed by the Chi square test or Fisher's exact test, the Student t-test or the Mann-Whitney test as appropriate.

Results were considered statistically significant for $p$-values $<0.05$. Stata software (StataCorp. 2019. Stata Statistical Software: Release 16. College Station, TX: StataCorp LLC) was used for statistical analysis.

\section{Results}

\section{Study groups}

The study groups were similar in terms of demographic characteristics, duration of operation and duration of anesthesia (Table 1). In the dexmedetomidine group, 24 patients received general anesthesia (dexmedetomidine group, general anesthesia) and 26 received a combined anesthesia (dexmedetomidine group, combined anesthesia). In the control group, 30 patients received general anesthesia (control group, general anesthesia) and 26 patients combined anesthesia (control group, combined anesthesia).

\section{Incidence of emergency delirium}

Figure 1 summarizes the incidence of ED in the different groups. Three patients who received dexmedetomidine premedication showed ED (6\%), compared with 43 patients in the control group (77\%; $p<0.05)$. This lower incidence of ED was also present when stratifying patients according to the type of anesthesia or age (Figure 1). In particular, no patient on dexmedetomidine receiving combined anesthesia or in the age groups $2-3$ years and 6-10 years presented ED.

\section{Time of anesthesia and time to hospital discharge}

We observed no relevant differences between the dexmedetomidine group and the control group in terms of timing of discharge from surgical area, regardless of the specific indication to plastic surgery (Table 2).

\section{Safety}


No relevant hemodynamic effects were observed in patients in the dexmedetomidine group compared with the control group. In fact, we did not observe any significant difference in the mean heart rate and mean arterial pressure at baseline, 15 and 30 minutes after dexmedetomidine intranasal administration (Table 3). No adverse events directly associated with dexmedetomidine administration were reported.

\section{Discussion}

The awareness of a state of agitation linked in some way to the postoperative phase and closely related to anesthesia has been described in literature as far back as the 1960s [25]. The etiology of postoperative ED remains unclear [26,27], but the introduction of modern short-acting volatile anesthetics has been associated with the increased incidence of ED, because of their interference with the balance between neuronal synaptic inhibition and excitation in the central nervous system [28].

Over the last few years, in attempt to reduce the risk of ED, prophylactic measures such as coadministration of propofol, midazolam or fentanyl were used, but the risks associated with their use was greater than the benefit [28]. Therefore, strategies such as premedication with ketamine, fentanyl and $₫ 2$ adrenoreceptor agonists has been demonstrated to prevent ED [3]. In a meta-analysis, Zhu et al. have showed that the intravenous intraoperative administration of dexmedetomidine can be effective in decreasing ED [17]. These authors analyzed recent literature and in their conclusion, they considered the opportunity to find out new strategies to avoid dexmedetomidine side-effects, such as the hemodynamic impact or the delay in recovery from anesthesia or in the time of staying in the surgical area [17].

In the present study, we have chosen to administer dexmedetomidine premedication intranasally, at the dose and waiting times supported by wider consensus [29], because this premedication is safe and well tolerated by patients [30]. In fact, usually most drug delivery in premedication requires an intravenous access. Although effective and associated with a fast onset of action, intravenous access is associated with pain and anxiety in children and may be difficult for inexperienced providers. Intranasal drug administration is an alternative method of medication delivery, it is painless, not associated with unpleasant sensation [31] and has a slower and more gradual onset than intravenous administration [32]. Moreover, this last characteristic is important in order to avoid $\otimes 2$-agonist side effects seen with intravenous administration, such as bradycardia and hypotension.

Remarkably, in our study, only a minority (6\%) of patients on dexmedetomidine experienced ED, compared with more than $75 \%$ of patients showing this event without dexmedetomidine administration. In some subgroups we also registered a very high occurrence of ED (e.g. 100\% in patients aged 3-6 years old, control group on general anesthesia), which was superior to some of the data reported in literature [33] and which might have been influenced by the limited number of patients in these groups. We believe that one of the main causes of ED in our population was the use of high dose volatile anesthetics, which are a known risk factor for ED occurrence $[1,28]$. Conversely, the type of surgery was probably a minor contributor to ED occurrence, given that patients underwent minor procedures lasting 60-90 minutes. 
We also evaluated the incidence of ED according to type of anesthesia (general or combined) or different ages: the advantage for dexmedetomidine was consistent across all these subgroups, and in those with an expected low rate of ED. In our data, in combined anesthesia and in ages over 6 years and under 3 years old, ED drops to $0 \%$ if premedication was performed, compared to, and, already low incidence of ED in these situations in controls (31\% over 6 years and $33 \%$ under 3 years). These data support the possible neuroprotective effect of dexmedetomidine that may help reduce the incidence of delirium in patients undergoing loco-regional anesthesia in which the occurrence of ED is usually not caused by uncontrolled pain. This finding is of major relevance, since the use of dexmedetomidine has been poorly explored to date in pediatric patients subjected to combined anesthesia. Moreover, this observation indirectly shows a relationship between regional anesthesia and reduced ED occurrence, which might be attributed to the decreased need for volatile anesthetic, a known risk factor for ED [28]. With respect to safety, our results are in line with previous reports $[12,30,34]$, since no patients who received dexmedetomidine intranasally in premedication had significant hemodynamic effects. Moreover, at the same time, the administration given in advance compared with the intravenous one can avoid the delay in recovery from anesthesia and in length of staying in surgical area [17]. In our study, no patients in the dexmedetomidine group had longer time of recovery from anesthesia or longer time of staying in recovery room compared with the control group; however, we acknowledge that the heterogeneity of surgical procedures and the limited number of patients belonging to each surgery type might have influenced this result.

This study presents several limitations, including all those inherent to any retrospective study (e.g., poor reporting and selection of treatment according to the clinical status of the patient, lack of blinding and randomization) and the fact that the stratification based on age and type of surgery has resulted in a limited number of patients included in each sub-group. Moreover, in order to enhance homogeneity, we considered only patients undergoing interventions with a relatively short surgical time (60-90 minutes), within the range of dexmedetomidine half-life. We acknowledge that some confounding factors may exist in this study, such as the lack of pre-medication in the control group and the fact that we did not perform a formal analysis of possible factors influencing ED occurrence, such as the level of pain in the recovery room or the requirement for rescue analgesia among patients' subgroups. With regards to pain, we acknowledge that in the recovery room is difficult to determine, especially in younger patients, if distress and inconsolable crying are caused by pain or should be attributed to ED. However, we believe that in our population the analgesic control was sufficient to limit the occurrence of pain, as assessed by the low VAS/FLACC scores (data not shown), and also considering that patients underwent minor surgical procedures lasting 60-90 minutes, a situation in which an adequate pain control can be obtained with general anesthesia.

\section{Conclusions}

Intranasal dexmedetomidine as a premedication seems to be a safe procedure that can be associated with decreased incidence of ED without increasing the timing of anesthesia or timing of staying in the recovery room after surgery, regardless of patients' age or type of anesthesia. In particular, 
dexmedetomidine seemed to be beneficial for patients undergoing combined anesthesia, who reported no incidence of ED, thus suggesting a possible neuroprotective role of this agent.

\section{Abbreviations}

ASA: American Society of Anesthesiologists

ED: emergency delirium

\section{Declarations}

\section{Ethics approval and consent to participate}

The Ethical Committee of the Bambin Gesù Hospital has approved the study design (protocol number 1587_OPBG_2018). The legal guardians of all patients signed an informed consent for the use of the patient's data for research purposes.

\section{Consent for publication}

Not applicable

\section{Availability of data and materials}

The datasets used and/or analyzed during the current study are available from the corresponding author on reasonable request.

\section{Competing interests}

Orion Pharma supported the editorial assistance of this manuscript. The authors declare no additional competing interests.

\section{Funding}

The study was funded by departmental resources.

\section{Authors' contributions}

Study conception and design: ADP, SGP 
Data collection: All

Data analysis and interpretation: All

Manuscript drafting: ADP, FM,

Manuscript editing: All

Approval to submit: All

\section{Acknowledgements}

The authors thank Luca Giacomelli (Polistudium srl) for his contribution in drafting the paper and revising it.

Editorial assistance was provided by Sara Di Nunzio and Aashni Shah (Polistudium srl, Milan, Italy). This assistance was supported by Orion Pharma.

\section{References}

1. Mason KP. Paediatric emergence delirium: a comprehensive review and interpretation of the literature. Br J Anaesth 2017;118(3): 335-343.

2. Moore AD, Anghelescu DL. Emergence delirium in pediatric anesthesia. Paediatr Drugs 2017;19(1):11-20. doi: 10.1007/s40272-016-0201-5.

3. Reduque LL, Verghese ST. Paediatric emergence delirium. Continuing Education in Anaesthesia Critical Care \& Pain 2013;13(2): 39-41.

4. Kain ZN, Mayes LC, Caldwell-Andrews AA, Karas DE, McClain BC. Preoperative anxiety, postoperative pain, and behavioral recovery in young children undergoing surgery. Pediatrics 2006;118(2): 651-8.

5. Vernon DT, Schulman JL, Foley JM. Changes in children's behavior after hospitalization. Some dimensions of response and their correlates. Am J Dis Child 1966;111(6): 581-93.

6. Kain ZN, Caldwell-Andrews AA, Maranets I, McClain B, Gaal D, Mayes LC, et al. Preoperative anxiety and emergence delirium and postoperative maladaptive behaviors. Anesth Analg 2004;99(6):164854.

7. Aono J, Ueda W, Mamiya K, Takimoto E, Manabe M. Greater incidence of delirium during recovery from sevoflurane anesthesia in preschool boys. Anesthesiology 1997;87(6): 1298-300.

8. Vlajkovic GP, Sindjelic RP. Emergence delirium in children: many questions, few answers. Anesth Analg 2007;104(1):84-91.

9. Voepel-Lewis T, Malviya S, Tait AR. A prospective cohort study of emergence agitation in the pediatric postanesthesia care unit. Anesth Analg 2003;96(6):1625-30. 
10. Jun JH, Kim KN, Kim JY, Song SM. The effects of intranasal dexmedetomidine premedication in children: a systematic review and meta-analysis. Can J Anaesth 2017;64(9):947-961.

11. Sottas CE, Anderson BJ. Dexmedetomidine: the new all-in-one drug in paediatric anaesthesia? Curr Opin Anaesthesiol 2017;30(4):441-451. doi: 10.1097/AC0.0000000000000488.

12. Uusalo P, Lehtinen M, Löyttyniemi E, Manner T, Scheinin M, Saari TI. Premedication with intranasal dexmedetomidine decreases barbiturate requirement in pediatric patients sedated for magnetic resonance imaging: a retrospective study. BMC Anesthesiol. 2019;19(1):22. doi: 10.1186/s12871019-0690-1.

13. FitzSimons J, Bonanno LS, Pierce S, Badeaux J. Effectiveness of preoperative intranasal dexmedetomidine, compared with oral midazolam, for the prevention of emergence delirium in the pediatric patient undergoing general anesthesia: a systematic review. JBI Database System Rev Implement Rep 2017;15(7):1934-1951.

14. Plambech $M Z$, Afshari A. Dexmedetomidine in the pediatric population: a review. Minerva Anestesiol 2015;81(3):320-32.

15. A practice of anesthesia for infants and children, 6th edition (Elsevier,2018) Cote' CJ, Lerman J, Anderson BJ. Cote' and Lerman's.

16. Yao Y, Qian B, Lin Y, Wu W, Ye H, Chen Y. Intranasal dexmedetomidine premedication reduces minimum alveolar concentration of sevoflurane for laryngeal mask airway insertion and emergence delirium in children: a prospective, randomized, double-blind, placebo-controlled trial. Paediatr Anaesth 2015;25(5):492-8.

17. Zhu M, Wang H, Zhu A, Niu K, Wang G. Meta-analysis of dexmedetomidine on emergence agitation and recovery profiles in children after sevorane anesthesia: different administration and different dosage. PLoS One 2015;10(4):e0123728.

18. Stamper MJ, Hawks SJ, Taicher BM, Bonta J, Brandon DH. Identifying pediatric emergence delirium by using the PAED Scale: a quality improvement project. AORN J 2014;99(4):480-94.

19. Sikich N. Development of psychometric evaluation of the Pediatric Anesthesia Emergency Delirium Scale. Anesthesiology 2004;100:1138-45.

20. Sethi S, Ghai B, Ram J, Wig J. Postoperative emergence delirium in pediatric patients undergoing cataract surgery-a comparison of desflurane and sevoflurane. Peaediatr Anaesth 2013;23(12):1131-37.

21. Aldrete JA. The post-anesthesia recovery score revisited. J Clin Anesth 1995;7(1):89-91.

22. Merkel SI, Voepel-Lewis T, Shayevitz JR, Malviya S. The FLACC: a behavioral scale for scoring postoperative pain in young children. Pediatr Nurs 1997;23: 293-97.

23. Yuen VM, Hui TW, Irwin MG, Yao TJ, Wong GL, Yuen MK. Optimal timing for the administration of intranasal dexmedetomidine for premedication in children. Anaesthesia 2010;65(9):922-929.

24. Xie Z, Shen W, Lin J, Xiao L, Liao M, Gan X. Sedation effects of intranasal dexmedetomidine delivered as sprays versus drops on pediatric response to venous cannulation. Am J Emerg Med 2017;35(8):1126-1130.

Page $11 / 16$ 
25. Smessaert A, Schehr CA, Artusio JF Jr. Observations in the immediate postanaesthesia period. II. Mode of recovery. Br J Anaesth 1960;32:181-5.

26. Koch S, Stegherr AM, Rupp L, Kruppa J, Prager C, Kramer S, et al. Emergence delirium in children is not related to intraoperative burst suppression-prospective, observational electrography study. BMC Anesthesiol. 2019 Aug 8;19(1):146. doi: 10.1186/s12871-019-0819-2.

27. Li LQ, Wang C, Xu HY, Lu HL, Zhang HZ. Effects of different doses of intranasal dexmedetomidine on preoperative sedation and postoperative agitation in pediatric with total intravenous anesthesia undergoing adenoidectomy with or without tonsillectomy. Medicine (Baltimore) 2018;97(39):e12140.

28. Vlajkovic GP, Sindjelic RP. Emergence delirium in children: many questions, few answers. Anesth Analg 2007;104(1):84-91.

29. Miller JW, Divanovic AA, Hossain MM, Mahmoud MA, Loepke AW. Dosing and efficacy of intranasal dexmedetomidine sedation for pediatric transthoracic echocardiography: a retrospective study. Can J Anaesth 2016;63(7): 834-41.

30. Lin $\mathrm{Y}$, Chen $\mathrm{Y}$, Huang J, Chen $\mathrm{H}$, Shen W, Guo W, et al. Efficiency of premedication with intranasal dexmedetomidine on inhalational induction and postoperative emergence agitation in pediatric undergoing cataract surgery with sevoflurane. J Clin Anesth 2016;33:289-95.

31. Wolfe TR, Braude DA. Intranasal medication delivery for children: a brief review and update. Pediatrics 2010;126(3):532-7.

32. Li A, Yuen VM, Goulay-Dufaÿ S, Sheng Y, Standing JF, Kwok PCL, Leung MKM, Leung AS, Wong ICK, Irwin MG. Pharmacokinetic and pharmacodynamic study of intranasal and intravenous dexmedetomidine. Br J Anaesth 2018;120(5):960-968.

33. Pickard A, Davies P, Birnie K, Beringer R.Systematic review and meta-analysis of the effect of intraoperative a $\mathbb{X}$-adrenergic agonists on postoperative behaviour in children. $\mathrm{Br} \mathrm{J}$ Anaesth 2014;112(6):982-90.

34. Boules NS, Hanna HZ. Premedication with dexmedetomidine decreases emergence agitation after sevoflurane anesthesia in children. Ains Shams J Anesthesiol 2014;7(3):340-345.

\section{Tables}

Table 1. Patients' characteristics. 


\begin{tabular}{|c|c|c|c|}
\hline Characteristics & $\begin{array}{l}\text { Dexmedetomidine group } \\
\qquad(\mathrm{n}=50)\end{array}$ & $\begin{array}{l}\text { Control group } \\
\qquad(n=56)\end{array}$ & $\begin{array}{c}\mathrm{p}- \\
\text { value }\end{array}$ \\
\hline Age (years), mean \pm SD & $5.32 \pm 4.68$ & $5.74 \pm 4.26$ & 0.315 \\
\hline \multicolumn{4}{|l|}{ Age distribution, $\mathrm{n}(\%)$ : } \\
\hline . $\quad<3$ years & $7(14)$ & $11(20)$ & \multirow[t]{3}{*}{0.417} \\
\hline . 3-6 years & $15(30)$ & $11(20)$ & \\
\hline . $>6$ years & $28(56)$ & $34(60)$ & \\
\hline Males, n (\%) & $33(66)$ & $38(76)$ & 0.839 \\
\hline \multicolumn{4}{|l|}{ Indication for surgery: } \\
\hline . Burns/skin lesions & $5(10)$ & $3(5)$ & \multirow[t]{5}{*}{0.708} \\
\hline - Vascular malformation & $7(14)$ & $8(14)$ & \\
\hline - Hypospadias & $5(10)$ & $9(16)$ & \\
\hline - Syndactyly/polydactyly & $7(14)$ & $5(9)$ & \\
\hline - Giant nevus & $26(52)$ & $31(56)$ & \\
\hline
\end{tabular}

p-values are calculated using Student T-Test or Pearson's Chi square, when appropriate.

Table 2. Comparison of timing of anesthesia of the dexmedetomidine group and the control group. 


\begin{tabular}{|c|c|c|c|c|c|c|}
\hline & \multicolumn{3}{|c|}{ Duration of anesthesia (minutes) } & \multicolumn{3}{|c|}{ Discharge time from operating area (minutes) } \\
\hline & $\begin{array}{c}\text { Dexmedetomidine } \\
\text { group, mean } \pm S D(95 \% \\
\text { CI) }\end{array}$ & $\begin{array}{c}\text { Control group, } \\
\text { mean } \pm S D(95 \% \\
\text { CI) }\end{array}$ & $\begin{array}{c}p- \\
\text { value }\end{array}$ & $\begin{array}{c}\text { Dexmedetomidine } \\
\text { group, mean } \pm S D(95 \% \\
\text { CI) }\end{array}$ & $\begin{array}{l}\text { Control group, } \\
\text { mean } \pm S D(95 \% \\
C I)\end{array}$ & $\begin{array}{c}p- \\
\text { value }\end{array}$ \\
\hline Giant nevus & $78 \pm 34(64.26-91.73)$ & $\begin{array}{c}68 \pm 23(59.56- \\
76.53)\end{array}$ & 0.20 & $96 \pm 14(90.34-101.65)$ & $\begin{array}{c}101 \pm 28(90.72- \\
111.27)\end{array}$ & 0.40 \\
\hline Vascular malformation & $66 \pm 15(52.12-79.87)$ & $\begin{array}{c}71 \pm 15(57.12- \\
84.87)\end{array}$ & 0.53 & $\begin{array}{c}104 \pm 25(80.87- \\
127.12)\end{array}$ & $\begin{array}{c}111 \pm 21(93.44- \\
128.55)\end{array}$ & 0.57 \\
\hline Hypospadia & $56 \pm 13(39.85-74.14)$ & $\begin{array}{c}50 \pm 17(36.93- \\
63.06)\end{array}$ & 0.51 & $98 \pm 21(71.92-124.07)$ & $\begin{array}{c}105 \pm 18(91.16- \\
118.83)\end{array}$ & 0.52 \\
\hline Burns and other lesions & $69 \pm 30(31.75-106.24)$ & $\begin{array}{c}73 \pm 27(5.92- \\
140.07)\end{array}$ & 0.85 & $\begin{array}{c}119 \pm 38(71.81- \\
166.18)\end{array}$ & $\begin{array}{c}124 \pm 19(76.80- \\
171.19)\end{array}$ & 0.81 \\
\hline Syndactilia/polydactilia & $71 \pm 26(46.95-95.04)$ & $\begin{array}{c}76 \pm 16(56.13- \\
95.86)\end{array}$ & 0.70 & $\begin{array}{c}101 \pm 26(76.95- \\
125.04)\end{array}$ & $\begin{array}{c}106 \pm 15(87.37- \\
124.07)\end{array}$ & 0.69 \\
\hline
\end{tabular}

p-values are calculated using Student's t-test.

Table 3. Heart rate and mean arterial pressure before premedication with intranasal dexmedetomidine, 15 minutes and 30 minutes after premedication of the three groups divided by age. 


\begin{tabular}{|c|c|c|c|c|c|c|c|c|}
\hline \multirow{2}{*}{$\begin{array}{c}\text { Age } \\
\text { (years) }\end{array}$} & \multicolumn{4}{|c|}{ Heart rate (beat/min) } & \multicolumn{4}{|c|}{ Mean arterial pressure $(\mathrm{mmHg})$} \\
\hline & $\begin{array}{c}\text { Before } \\
\text { premedication, } \\
\text { mean } \pm S D \\
(95 \% C l)\end{array}$ & $\begin{array}{c}15 \text { min after } \\
\text { premedication, } \\
\text { mean } \pm S D(95 \% \\
\text { CI) }\end{array}$ & $\begin{array}{c}30 \text { min after } \\
\text { premedication, } \\
\text { mean } \pm S D(95 \% \\
C I)\end{array}$ & $\begin{array}{c}p- \\
\text { value }\end{array}$ & $\begin{array}{c}\text { Before } \\
\text { premedication, } \\
\text { mean } \pm S D \\
(95 \% \mathrm{Cl})\end{array}$ & $\begin{array}{c}15 \text { min after } \\
\text { premedication, } \\
\text { mean } \pm S D(95 \% \\
\text { CI) }\end{array}$ & $\begin{array}{c}30 \text { min after } \\
\text { premedication, } \\
\text { mean } \pm S D(95 \% \\
C I)\end{array}$ & $\begin{array}{c}p- \\
\text { value }\end{array}$ \\
\hline$<3$ & $\begin{array}{c}100 \pm 8(92.60- \\
107.39)\end{array}$ & $\begin{array}{c}97 \pm 7(90.52- \\
103.47)\end{array}$ & $\begin{array}{c}96 \pm 6(90.45- \\
101.54)\end{array}$ & 0.25 & $\begin{array}{c}73 \pm 2(71.15- \\
74.84)\end{array}$ & $\begin{array}{c}72 \pm 1(71.07- \\
72.92)\end{array}$ & $\begin{array}{c}71 \pm 1 \text { (70.07- } \\
71.92)\end{array}$ & 0.34 \\
\hline $3-6$ & $\begin{array}{c}95 \pm 6(91.67- \\
98.32)\end{array}$ & $\begin{array}{c}93 \pm 7(89.12- \\
96.87)\end{array}$ & $\begin{array}{c}92 \pm 7(88.12- \\
95.87)\end{array}$ & 0.67 & $\begin{array}{c}75 \pm 3(73.33- \\
76.66)\end{array}$ & $\begin{array}{c}74 \pm 2(72.89- \\
75.10)\end{array}$ & $\begin{array}{c}73 \pm 2(71.89- \\
74.10)\end{array}$ & 0.56 \\
\hline$>6$ & $\begin{array}{c}88 \pm 8(84.89- \\
91.10)\end{array}$ & $\begin{array}{c}86 \pm 8(82.89- \\
89.10)\end{array}$ & $\begin{array}{c}85 \pm 9(81.51- \\
88.48)\end{array}$ & 0.56 & $\begin{array}{c}81 \pm 3(79.83- \\
82.16)\end{array}$ & $\begin{array}{c}80 \pm 3(78.83- \\
81.16)\end{array}$ & $\begin{array}{c}79 \pm 1(78.61- \\
79.38)\end{array}$ & 0.41 \\
\hline
\end{tabular}

p-values are calculated using Student's t-test.

Figures 


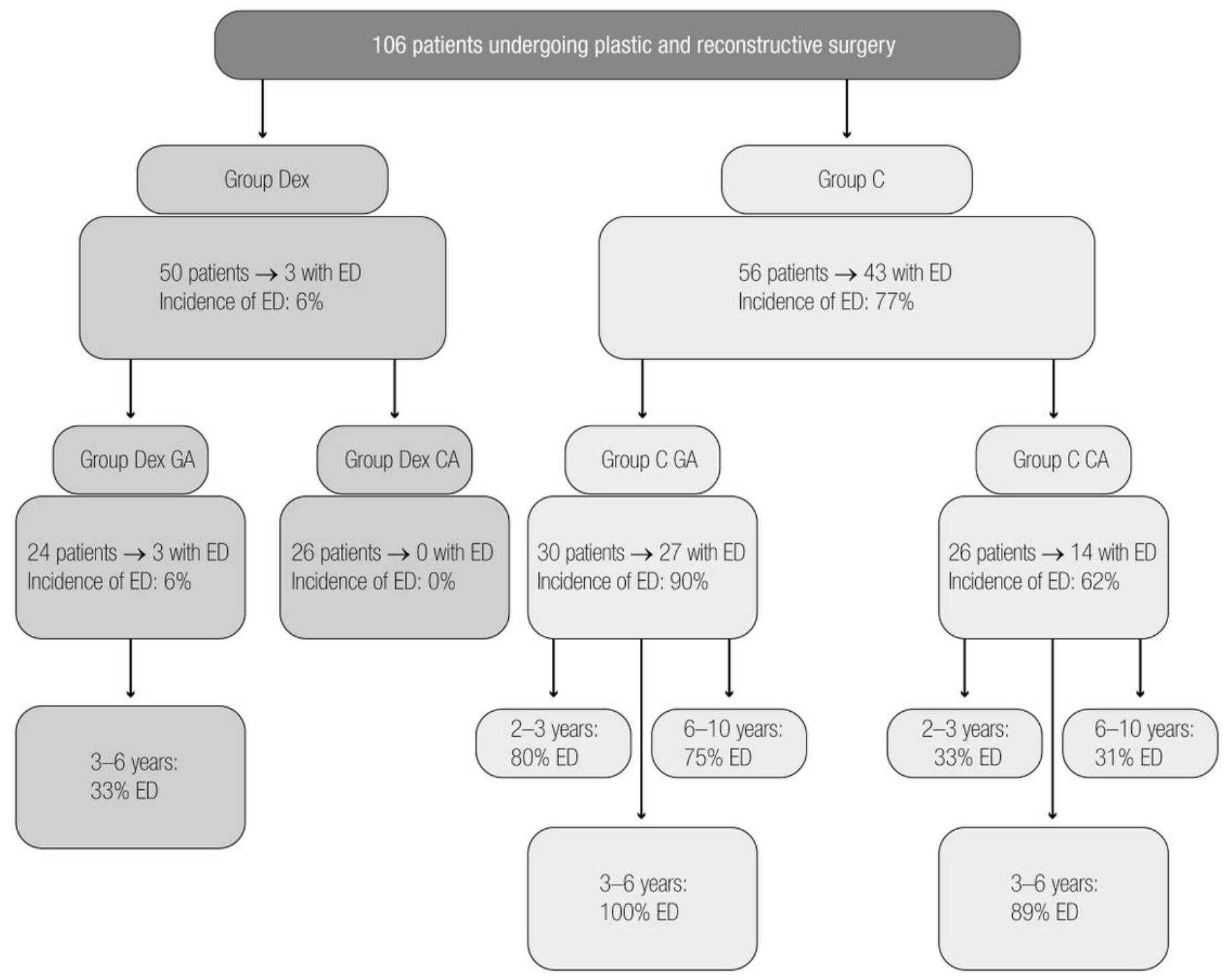

Figure 1

Distribution of emergency delirium in the study groups. CA: combined anesthesia; ED: emergency delirium; GA: general anesthesia. 